\title{
Cooperative motion of intrinsic and actuated semiflexible swimmers
}

\author{
I. Llopis \\ Departament de Física i Enginyeria Nuclear, Universitat Politècnica de Catalunya, Campus del Baix Llobregat, C. Esteve Terradas 8, 08860 \\ Castelldefels, Barcelona, Spain and Unitat de Tuberculosi Experimental, Fundació Institut per a la Investigació en Ciències de la Salut \\ Germans Trias i Pujol, Universitat Autònoma de Barcelona, Carretera de Can Ruti, Camí de les Escoles, \\ s/n, 08916 Badalona, Barcelona, Spain \\ I. Pagonabarraga \\ Departament de Física Fonamental, Universitat de Barcelona, C. Martí i Franqués 1, 08028 Barcelona, Spain \\ M. Cosentino Lagomarsino \\ Genomic Physics Group, UMR No. 7238, CNRS, “Microorganism Genomics,” and University Pierre et Marie Curie, 15 rue de l'École de \\ Médecine Paris, France and Dipartimento di Fisica, Universitá di Torino, via P. Giuria 1, Torino, Italy \\ C. P. Lowe \\ Van't Hoff Institute for Molecular Science, University of Amsterdam, Nieuwe Achtergracht 188, 1018 WV Amsterdam, The Netherlands
}

(Received 18 September 2012; published 25 March 2013)

\begin{abstract}
We examine the phenomenon of hydrodynamic-induced cooperativity for pairs of flagellated micro-organism swimmers, of which spermatozoa cells are an example. We consider semiflexible swimmers, where inextensible filaments are driven by an internal intrinsic force and torque-free mechanism (intrinsic swimmers). The velocity gain for swimming cooperatively, which depends on both the geometry and the driving, develops as a result of the near-field coupling of bending and hydrodynamic stresses. We identify the regimes where hydrodynamic cooperativity is advantageous and quantify the change in efficiency. When the filaments' axes are parallel, hydrodynamic interaction induces a directional instability that causes semiflexible swimmers that profit from swimming together to move apart from each other. Biologically, this implies that flagella need to select different synchronized collective states and to compensate for directional instabilities (e.g., by binding) in order to profit from swimming together. By analyzing the cooperative motion of pairs of externally actuated filaments, we assess the impact that stress distribution along the filaments has on their collective displacements.
\end{abstract}

DOI: 10.1103/PhysRevE.87.032720

PACS number(s): 87.17.Aa, 82.70.Dd, 47.63.Gd, 47.63.mf

\section{INTRODUCTION}

Eukaryotic flagella are the engine for the fluid-based locomotion of a variety of microscopic animal, plant, and protist cells. These internally driven flexible microfilaments extend from the cell body and generate a wavelike motion that can propel the surrounding fluid [1]. The flow generated allows flagella to swim at low Reynolds numbers, where inertia is negligible. This is the regime characteristic of small-scale swimming [2]. At these scales, the flow induced in the embedding medium also provides a source of long-range dynamic interactions between different flagella. Recent experimental advances allow quantitative analysis of the hydrodynamic flows induced by swimming microscopic objects [3].

Starting from the observations made by Taylor [4], the mechanisms controlling flagellar swimming have continued to attract the attention of physicists [4-7]. Since the internal drive of a flagellum is complex, a few relatively recent studies have focused on simplified model microswimmers, proposed to classify swimming strategies. These simple experimental and theoretical swimming models are more easy to approach both analytically and computationally and have allowed progress in the understanding of the mechanisms underlying the efficiency and velocity of flagellar propulsion [8-11]. Such models, referred to as semiflexible swimmers, are a special case of microswimmers for which the reciprocity is broken by filament flexibility. In most of these models, the configurational changes, which develop as a result of the competition between the forcing stresses and the restoring bending forces, induce a wave along the filament breaking reciprocity [2] and allowing propulsion in the absence of inertia.

Additionally, flagella have evolved in conditions where fluid-mediated dynamic interactions induced by nearby swimmers might be relevant. We refer, for example, to the situation of spermatozoa [12] of unicellular algae [13]. Do cells exploit the interactions for faster or more efficient swimming? While spermatozoa competition is a well established concept for spermatozoon cells, hydrodynamic interactions could also lead to cell cooperativity. As a matter of fact, for some murine rodent spermatozoa, this factor is known to exist [14]. These cells are characterized by a crescent-shaped head with a hook of varying size. They possess hooks that link the spermatozoon cells together in trains, allowing them to reach larger velocities and efficiencies, especially in viscous media [15]. The existence of a hook, which keeps the cells together, poses the question of whether dynamic interactions affect the directional stability of individual cells. These must maintain a common directionality, without being dispersed by the presence of nearby swimmers, and give them direction [16].

Hence the advantage of cooperativity can be seen as complementary to other known aspects of collective swimming, such as synchronization, observed in eukaryotic flagella 
[17-19]. Hydrodynamic interactions can lead to synchronization of model one-tailed swimmers [20,21]. This mechanism may promote collective motion through phase locking, as observed in bull spermatozoa [22], as well as the development of metachronal waves of cilia in micro-organisms [23-25].

To focus on the generic physical features of flagellar swimming, we will concentrate on the basic hydrodynamic mechanisms leading to cooperative motion of a pair of semiflexible swimmers. Although the detail of the internal force-generation mechanism will be neglected, the simplified driving force that we will consider emerges naturally from more detailed models that analyze flagellar internal propulsion [26]. While different models for internal propulsion are possible [10,26,27], selecting a simple description of the stresses that drive the flagella greatly simplifies the study of the interplay between elasticity and hydrodynamic forces controlling the cooperative motion of flagella. We will show how hydrodynamic interactions between filaments affect their collective motion and that the combination of elastic deformation and transverse beating provides specific features that cannot be captured by simpler swimmer models.

We mainly analyze the cooperative swimming of model flagella operated through an active internal mechanism, named intrinsic swimmers. This mechanism that gives rise to propulsion is force-free and intrinsic to the micro-organism itself. To highlight the peculiar features associated with the intrinsic swimming mechanism of semiflexible swimmers, we will also briefly discuss the cooperative swimming of passive externally actuated filaments, referred to as actuated swimmers or one-armed swimmers [8]. These actuated swimmers are technologically relevant because today's technology allows the realization of robotic microswimmers [28-30]. Actuated by external magnetic or electric fields, they are candidates for targeted drug delivery. In these actuated swimmers, a net instantaneous external force and/or torque is needed to generate motion, while for biological flagella and intrinsic swimmers in general, the internal mechanism producing a stress along the appendage is always force- and torque-free. These two different situations are dynamically distinct [31,32] and may be characterized by qualitatively different cooperative mechanisms. We will show that the specific driving affects qualitatively the hydrodynamic cooperativity of nearby semiflexible swimmers.

The rest of this work is organized as follows. In Sec. II we introduce the model for the semiflexible swimmer and the main features of single-swimmer dynamics. Section III discusses the main features of the cooperative motion of a pair of intrinsic microswimmers. Section IV highlights the main differences associated with actuated swimmers and Sec. V analyzes the efficiency of intrinsic cooperative swimming. We conclude in Sec. VI with a discussion of the main results obtained and their implications.

\section{MODEL AND BACKGROUND}

Here we recapitulate the main elements of the model and the main features of the motion of isolated intrinsic and actuated semiflexible swimmers. The filament is represented as an $N$ bead chain [33], where beads are connected by bonds of length $b$, so that every filament has a constant length $L \equiv(N-1) b$.
The bead size $a$ is fixed for all beads as $a=b / 2$, following the standard procedure in polymer physics $[34,35]$. The sizes and velocities of such filaments are such that inertia is negligible. Even though in this approach beads have a certain mass, in practice it is chosen to be sufficiently small $[33,36]$.

Semiflexibility is enforced by accounting for the bending energy between the beads along the filament. Consistent with the discretized description of the flagellum, the bending energy is expressed as

$$
\mathcal{H}_{B}=\frac{1}{2} \kappa b \sum_{i=2}^{N-1} C_{i}^{2}=\frac{1}{2} \kappa b \sum_{i=2}^{N-1} \frac{2}{b}\left(1-\cos \theta_{i}\right),
$$

where $\kappa$ is the filament stiffness and $C_{i}$ is the local curvature at bead $i$. The second equality provides an explicit expression of such a curvature as a function of $\theta_{i}$, the angle formed by the bond that connects bead $i-1$ to bead $i$ and the one that connects bead $i$ with bead $i+1$. The bending force on bead $i$ is obtained as the variation of $\mathcal{H}_{B}$ when bead $i$ changes its position [37].

The frictional force induced by the solvent is described as the difference between the bead velocity and the velocity $\mathbf{v}^{H}$ induced by the rest of the beads at the position of the bead of interest, namely,

$$
\mathbf{F}_{i}^{F}=-\gamma_{0}\left(\mathbf{v}_{i}-\mathbf{v}_{i}^{H}\right),
$$

where $\gamma_{0}$ is a reference friction coefficient (equal for all beads) and the Stokes expression $\gamma_{0}=6 \pi \eta a$ ( $\eta$ being the solvent viscosity) is used for simplicity. A tensorial friction coefficient slightly improves the model's quantitative predictive capabilities [36]. However, since we consider simplified model filaments, we consider it unnecessary here. The hydrodynamic velocity is computed within the Oseen approximation [38]

$$
\mathbf{v}_{i}^{H}(t)=\gamma_{0}^{-1} \frac{3}{4} \frac{a}{b} \sum_{j \neq i} \frac{1+\hat{r}_{i j}(t) \hat{r}_{i j}(t)}{r_{i j}(t) / b} \cdot \mathbf{F}_{j}(t),
$$

with the force $\mathbf{F}_{j}(t)$ corresponding to the total force acting on bead $j$ at time $t$. Note that this description does not include a model of the body of a swimming micro-organism, which could be implemented by adding a larger or less mobile bead at one extremity. A further simplified treatment of the interaction between the filament and the solvent (resistive force theory) disregards the flow induced by the filament itself and accordingly sets $\mathbf{v}_{i}^{H}=0$ [39]. This approach can describe the coupling between bending deformation in the nonreciprocal motion of a filament $[8,40]$, but fails to capture the hydrodynamic coupling and its effects in the collective motion of filaments. It is insufficient to study the emergence of hydrodynamic cooperativity.

We consider two very simple and qualitatively different drives leading to semiflexible filament swimming, corresponding to intrinsic and actuated swimmers. The former mimics more closely the dynamics of biological flagella, while the latter models externally driven filaments. Although we will focus on intrinsic swimmers, it is instructive to highlight the basic differences between the driving models.

\section{A. Intrinsic swimmer driving}

Disregarding the detailed mechanisms that propel a neutrally buoyant flagellum, we apply an intrinsic traveling 
force-quadrupole wave along the filament. Specifically, we consider a planar sinusoidal wave, propagating in a specified direction at constant speed. Such an approximation produces a planar distortion of the filament, similar to the waveforms of some spermatozoa [33]. This force distribution ensures that at any time there is no net force or torque, in accordance with Newton's third law. The force quadrupole is generated by pair of torques of magnitude $T_{i}$ acting on consecutive bonds with opposite sign. For every filament bead $i$ extending along the filament,

$$
T_{i}=T_{0} \sin \left[\frac{2 \pi}{\lambda} x_{i}-\frac{2 \pi}{\lambda} c t+\phi\right],
$$

where $x_{i}$ is the position of the $i$ th bond along the chain, $c$ is the wave propagation speed, $\phi$ is the initial value of the phase of the torque, and $\Omega=2 \pi c / \lambda$ is the driving frequency, with $\lambda$ the wavelength. In the numerical implementation we assume that the torque acts midway between consecutive beads and apply, accordingly, opposite forces with the magnitude of the bond torque in consecutive beads [33].

Although, in general, the motion of an intrinsic swimmer exhibits intrinsic self-rotation, which can lead to chiral sensing [41], we have minimized the effect by appropriately choosing $\lambda=L$. We have compared the swimming behavior at different values of $\lambda$ and verified that the results we report do not change qualitatively due to this effect.

\section{B. Actuated swimmer driving}

Different models of actuated artificial swimmers have been analyzed [29,42-44]. Here, motivated by its simplicity and because it constitutes a simple model for artificial swimmers [28,30], we will consider an actuated swimmer driven by a periodic force applied at one extreme [8,9]. It acts perpendicularly to the swimmer direction of motion with a magnitude

$$
F=F_{0} \cos (\Omega t+\phi) .
$$

Here $F_{0}$ is the external force amplitude, $\Omega$ is its frequency, and $\phi$ characterizes the initial state of this external forcing. As opposed to intrinsic swimmers, the driving force is now localized only on the first bead and the total force acting on an actuated swimmer vanishes only on average during one beating period. Actuated swimmers are therefore subject to instantaneous net accelerations induced by the applied force.

Both driving mechanisms give rise to cyclic nonreciprocal motion leading to filament propulsion. This arises purely from a coupling between the bending resistance of the filament and the anisotropic net drag imposed by the fluid environment due to the nonsymmetric shape of the chain over time [45].

\section{Dynamic evolution of single semiflexible swimmers}

We consider the numerical solution of the evolution equation for swimmers through a molecular dynamics algorithm implementing a velocity Verlet scheme. The internal tensions associated with filament inextensibility are treated as additional, constraining forces, using the efficient scheme proposed in Ref. [46]. A proper description of the forces acting on the filament requires a dynamical analysis of the filament acceleration. However, the inertial time scale is irrelevant in the motion of filaments at small Reynolds numbers. We ensure that this is the case by setting the time scale in which the filament adjusts to the flow velocity $m / \gamma_{0}$ much smaller than the characteristic times associated with shape distortions (which involve all the beads in the chain).

The parameter that characterizes the swimmer motion, for a given magnitude of driving, is the dimensionless number $\mathrm{Sp}$, or sperm number [33]. It quantifies the relative importance of viscous and elastic stresses on the filament

$$
\mathrm{Sp}=\left(\frac{L^{4} \Omega \xi_{\perp}}{\kappa}\right)^{1 / 4},
$$

where $\xi_{\perp}$ is the average transverse friction coefficient arising from the hydrodynamic interactions introduced through the viscous drag [9].

Figure 1 displays the swimming velocity of a single intrinsic and actuated swimmer at small driving amplitudes $F_{0}$ as a function of $\mathrm{Sp}$. The value of $\mathrm{Sp}$ at which optimal speed is achieved depends on the driving mechanism controlling
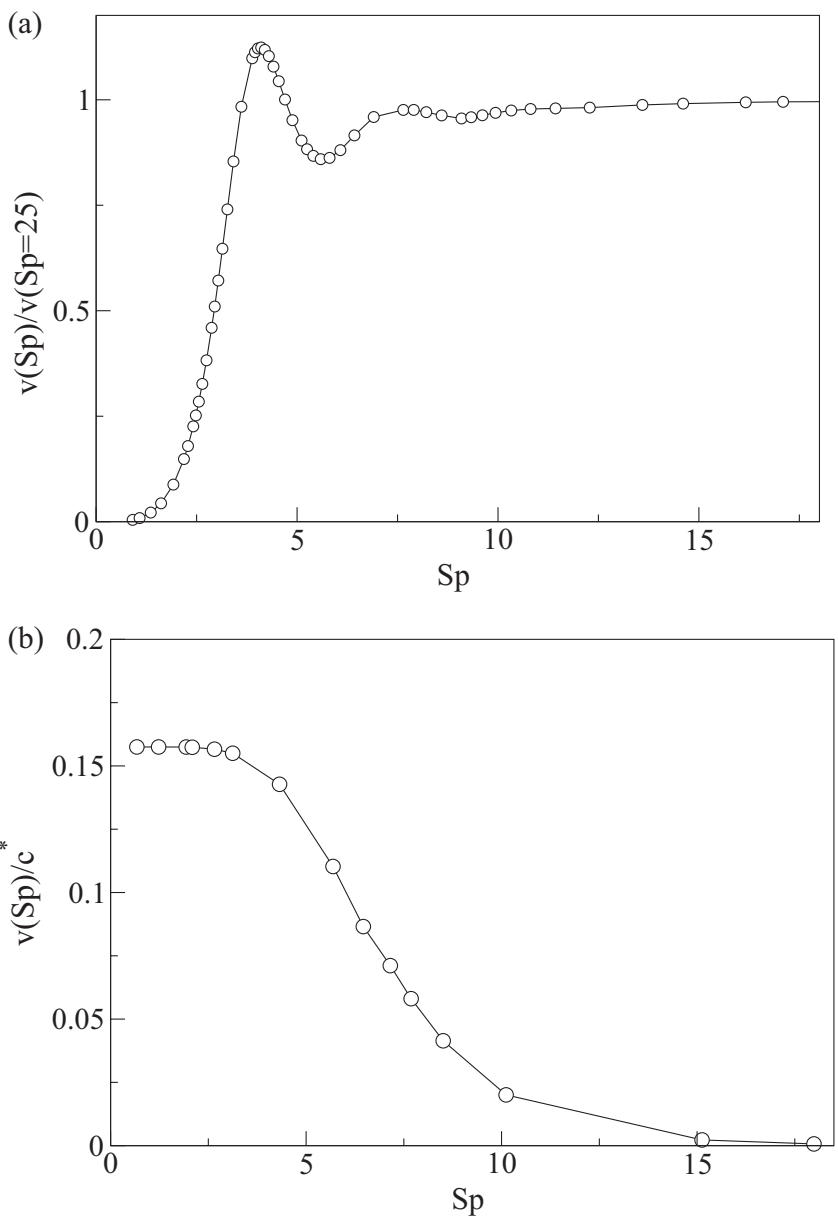

FIG. 1. Swimming velocity of a driven semiflexible filament as a function of $\mathrm{Sp}$ for the two different driving mechanisms: (a) actuated swimmer and (b) intrinsic swimmer. For an actuated swimmer, the speed is normalized by its asymptotic value at large $\mathrm{Sp}$, whereas for an intrinsic swimmer the apparent speed of the quadrupole wave $c^{*}=c L^{*} / L$ is introduced, where $L^{*}$ is the apparent length, i.e., the projection of the filament length $L$ on the propulsion direction; this magnitude decreases for larger $\mathrm{Sp}$. 
filament displacement. For example, for an actuated swimmer, an optimal swimming speed is achieved at $\mathrm{Sp} \sim 4$ [8,9]; for smaller $\mathrm{Sp}$ a rapid decrease with respect to the optimal value is observed as the filament becomes stiffer and for larger $\mathrm{Sp}$ it decreases slightly until it reaches a constant plateau value, as shown in Fig. 1(a). Upon increasing $F_{0}$, the velocity at large Sp decays to zero [9]. In actuated swimmers, the velocity decreases to zero at large Sp because of excessive drag on the filament [31]. For a pivoting prosthesis $S p \sim 2$, while for alternative models of self-propelled semiflexible filament swimmers optimal $\mathrm{Sp}>4$ have also been reported [47].

For intrinsic swimmers, the dependence of the propulsive velocity on $\mathrm{Sp}$ differs considerably from the one described for actuated ones. At small values of $\mathrm{Sp}$ the average velocity develops a plateau where the speed is maximal. Conversely, at large $\mathrm{Sp}$, the velocity decreases to zero, independent of the torque amplitude $T_{0}$ [33], as Fig. 1(b) shows, in agreement with the arguments presented in Ref. [31]. In all simulations we have discretized the chain into $N=30$ beads and have tuned the magnitude of the applied force $F_{0}$ and torque $T_{0}$ for a given value of the monomer friction coefficient $\xi_{\perp}$ and wave speed $c$ in such a way that in one beating period the first bead of the filament displaces one-tenth of the interbead distance. The beating frequency is always chosen to ensure an appropriate time-scale separation and that bead inertia is negligible [36].

\section{Arrangements of swimmer pairs}

To understand the generic effects that hydrodynamic interactions induce in the dynamic state of two identical swimmers, we concentrate on, and compare the outcome of, a few simple spatial arrangements. These are depicted in Fig. 2. The interactions between filaments depend both on the force that flagella impose on each other through the fluid and on their relative spatial configuration. To analyze the effect of these different contributions, we restrict ourselves to parallel filaments, separated by a distance $d$ perpendicular to the initial filament swimming direction. We then distinguish between coplanar swimmers (C) [see Fig. 2(a)] and transversal swimmers (T) [see Fig. 2(b)]. For completeness, we also briefly analyze the particular configuration of frontal swimmers $(F)$. Here the two filaments are separated by a distance $d$ along the same line, defined by the initial direction of swimming [Fig. 2(c)]. The driving force or torque acting on both filaments differs only in relative phase. The effect of this phase difference is illustrated by comparing the extreme cases where the two swimmers beat either in phase (P) $\phi_{1}=\phi_{2}$ or in antiphase (A) $\phi_{1}=\phi_{2}+\pi$.

\section{COOPERATIVE MOTION OF INTRINSICALLY DRIVEN FILAMENT SWIMMERS}

\section{A. Effects on swimmers' speed and deformation}

Semiflexible swimmers can either cooperate positively or hinder each other due to hydrodynamic interactions and the effect of this cooperation emerges as a balance between hydrodynamic and bending stresses. At distances long compared to the filament length $d \gg L$, the neighboring swimmer is felt dynamically as an oscillating quadrupole that perturbs the flow field. However, as we will see, such a simplified picture does not capture the main features of cooperative swimming at shorter separations. Nevertheless, it is useful to take advantage of the fact that, since the filaments feel each other asymptotically as oscillating quadrupoles, their coupling decays algebraically at long separations as $d^{-3}$. Furthermore, the filaments' relative phase and position determine whether the two swimmers hinder or promote their motion. The asymptotic decay of the flow field induced by the semiflexible swimmers depends on the specific nature of the forcing that controls their swimming. In the model swimmers we analyze the torque wave does not break fore-aft symmetry, hence leading to a velocity field that decays asymptotically as a quadrupole. In a more systematic analysis of simpler model swimmers it is possible to identify under which conditions the instantaneous induced velocity profile has a dipolar or quadrupolar nature [48,49].

\section{Mean filament velocity}

As shown in Fig. 3, two intrinsic swimmers swim faster with decreasing distance for antiphase beating, while a decrease in speed for decreasing distance is observed for in-phase beating. This is true for both coplanar and transverse geometries. However, the two geometries display qualitatively different velocity changes as a function of filament separation. Coplanar swimmers change their swimming speed monotonically as they approach. For swimmers beating in parallel planes, in contrast, in-phase swimming is more advantageous in terms of speed once filaments approach closer than approximately $d=0.3 L$. The converse is true when swimming in antiphase [Figs. 3(a) and 3(b)].
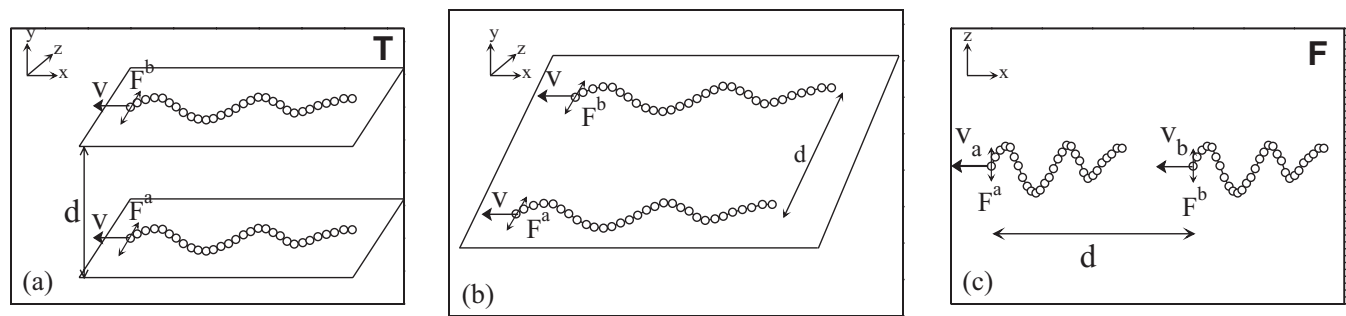

FIG. 2. Sketch of the geometry of the three initial semiflexible swimmer configurations analyzed here: (a) transverse swimmers (T), (b) coplanar swimmers (C), and (c) frontal swimmers (F). In the simulations, the filaments are prepared by swimming separately until they reach a steady state. In configurations $\mathrm{T}$ and $\mathrm{C}$ the initial swimming velocity of the two swimmers is the same, $v$, while the initial velocities of frontal swimmers do not have to coincide. 

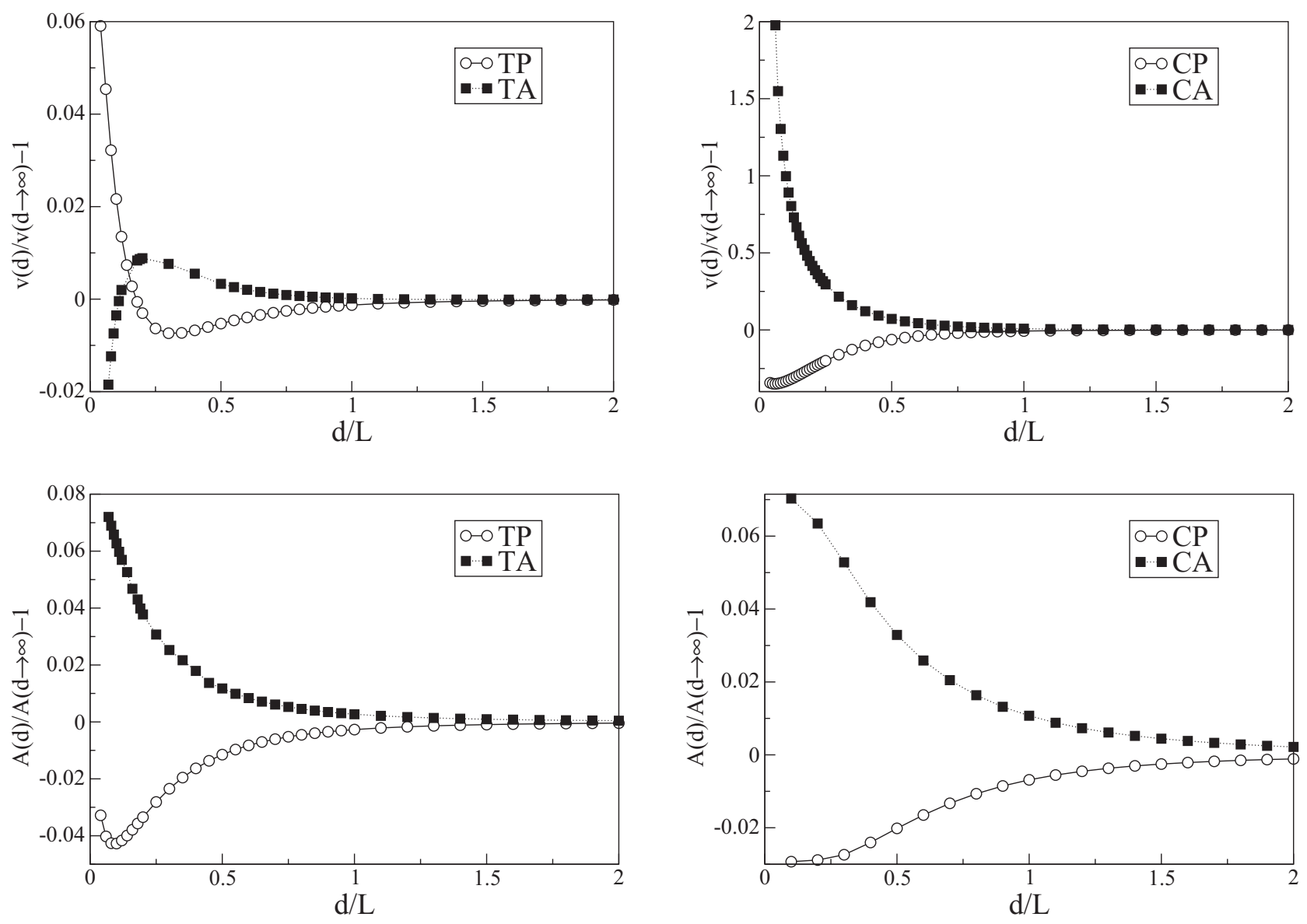

FIG. 3. Velocity (top panels) and amplitude (bottom panels) as a function of filament separation $d$ (rescaled by filament length), for $\mathrm{Sp}=2.26$, when intrinsic swimmers move in parallel planes (geometry $\mathrm{T}$, left) and in the same plane (geometry $\mathrm{C}$, right). In the legends, the labels $\mathrm{T}$ and $\mathrm{C}$ stand for the geometries described in Fig. 2 and the labels $\mathrm{A}$ and $\mathrm{P}$ for antiphase and in-phase, respectively. For the parameters used in the plot, $A(d \rightarrow \infty)=0.01 L$, but the results are equivalent for higher amplitudes.

\section{Beating amplitude}

Mean (longitudinal) swimming is distinct from the (transverse) beads' beating velocity resulting from the internal drive. The latter can be quantified by the maximum amplitude of the bead displacement in the chain $A$. This decreases monotonically as the distance decreases for in-phase beating, while the opposite is observed for antiphase beating, as shown in Figs. 3(c) and 3(d). Since the magnitude of the driving force is constant, an increase in the beating velocity must occur for the in-phase motion (and a decrease for antiphase motion). While for coplanar beating the amplitude follows the same trend at large distances, an extreme in the amplitude is reached at very small separation. This is not surprising: If two particles move in phase, the induced flow fields add constructively, leading to an increase of the filament velocity; the opposite is true for antiphase motion.

In contrast, it is surprising that while the hydrodynamic coupling is constructive in the beating direction, it can have the opposite effect in the transverse direction. This can be rationalized by the following considerations. We have verified that the average flow field induced by one swimmer on its neighbor, along the direction of motion, can reverse its direction as a result of the competition of hydrodynamic forces and tensions induced by bending. This emerges as a result of the coupling of local velocity flows with regions of the chain moving in opposite directions. Hence the net gain or loss induced by a neighboring swimmer has a complex dependence on both velocity and distance. Such couplings are intrinsic to the transverse character of the filament motion and cannot be recovered for simpler models of straight swimmers that deform only along their own axis [11,50]. The trend is observed independently of the value of $\mathrm{Sp}$; all else being equal, the more flexible the filament, the larger the velocity change due to the neighboring swimmer. Moreover, this result is robust with respect to the intrinsic amplitude of the driving mechanism; for the results reported, the amplitude was small $A(d \rightarrow \infty)=0.01 L$.

Even if the flow generated by the filaments is three dimensional, the peristaltic motion induced by antiphase beating promotes fluid flow and helps propel the filaments. This clearly indicates that the transverse deformation of the filament plays an important role in its hydrodynamic coupling. Comparing coplanar and transverse beating, one can appreciate that the induced flow heterogeneities in the former geometry are felt more directly by the filament conformational changes than in the latter. As a result, coplanar beating displays a stronger hydrodynamic coupling leading to a much more 


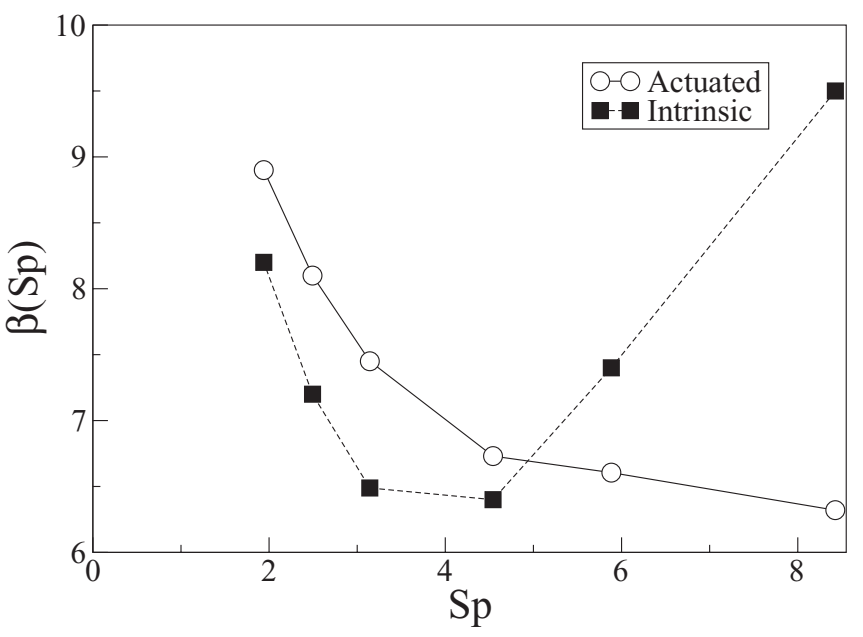

FIG. 4. Characteristic hydrodynamic interaction length for nearby semiflexible swimmers, $\beta$ (in units of bead size), as a function of $\mathrm{Sp}$, showing a different dependence for intrinsic and actuated swimmers. Here $A(d \rightarrow \infty)=0.01 L$.

marked hindrance or increase, in both the mean velocity and beating amplitude.

\section{Nearby semiflexible swimmers}

As opposed to the algebraic decay of the filament velocity for distant filaments, the interaction between nearby filaments can be described approximately as an exponential decay of the velocity with filament separation. By analyzing the decay of the velocity field as a function of the filament distance, we can extract an effective decay length $\beta$ for pairs of swimming flagella for a typical separation of the filament pair $d / L<0.25$. Figure 4 displays the dependence of the associated decay length $\beta$ on filament flexibility. The more flexible the flagellum, the less efficiently it can transmit the induced fluid flows leading to a faster velocity decay. This is consistent with the fact that cooperation requires that bending couples to the induced hydrodynamic stresses.

\section{In-line swimmers}

Cooperative swimming through hydrodynamic coupling is robust and the basic features described previously are found in generic swimmer configurations. For example, in Fig. 5 we display the relative velocity between the front and back filaments for frontal swimming. This plot shows that, counterintuitively, the frontal flagellum swims faster (the relative velocity $v_{r}$ is positive), giving rise to an effective repulsion between both swimmers for all separations. The asymptotic algebraic decay of the relative velocity $v_{r}$ maintains the expected dependence $v_{r}(d) \sim d^{-3}$.

\section{B. Interacting filaments are directionally unstable}

The hydrodynamic coupling of cooperative swimming leads also to a lateral interaction between filaments. This induces generically a relative nonzero lateral velocity, except for the particular geometry of frontal swimming. To assess the relevance of this coupling for directional instabilities, we consider the mean chain orientation of the filament as a

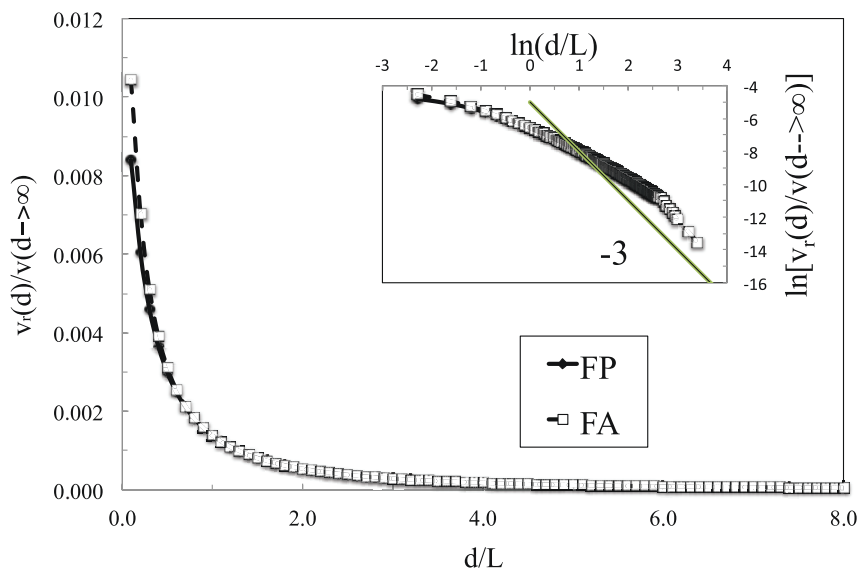

FIG. 5. (Color online) Relative velocity $v_{r}$ of frontal intrinsic microswimmers as a function of the minimum distance between them. As $v_{r}>0$, there is repulsion at initial times in this configuration. Here $d$ is the minimum distance between beads of different filaments, $A(d \rightarrow \infty)=0.01 L$, and $\mathrm{Sp}=2.26$. In the inset we depict the same on the log-log scale, showing the algebraic decay at large distances $v_{r} \sim d^{-3}$.

function of time. The ratio between the components of the filament velocity perpendicular and parallel to the flagellum orientation define the tangent of an angle $\theta$. This corresponds to the rate of change of the trajectory each swimmer describes with respect to its intrinsic displacement orientation. Since the filaments are deformable, alternative quantities can be considered to describe the degree of directional change of a flagellum. We have verified that other measures, such as the angle the mean filament orientation makes with the initial orientation (where the two filaments are parallel), do not change the qualitative behavior we describe. Due to filament deformation, the mean filament orientation, the direction of motion, and the tangent to the trajectory do not coincide.

Filaments start turning as soon as they interact with each other. However, turning takes place on a characteristic time $\tau_{c}=L \xi_{\perp} / T_{0}$ associated with the configurational relaxation of the filaments, with $\xi_{\perp}=4 \pi \eta L / \ln (L / b)$ the transverse friction coefficient of a rigid rod. This time is longer than that for which the swimmer can be displaced over its own length. Accordingly, we can distinguish between a short-time regime, in which filaments rotate without substantially modifying their initial relative position at a rather constant angular velocity $\omega$, and a second long-time regime, in which they approach or repel as a result of this angular velocity. The calculated translational velocities discussed previously correspond to this short-time regime, where filaments do not significantly change their relative separation.

\section{Short-time turning}

Figure 6 shows that initially two parallel intrinsic swimmers always attract each other and the more flexible, the filament the larger their turning velocity. Hence flexibility has a large impact on the relative approaching rate. As a result, for faster swimming, corresponding to intermediate and small $\mathrm{Sp}$, the initial filament configuration destabilizes more slowly. In general, there is an optimal distance, smaller than the flagellum size, at which the rate of rotation is maximal. In 

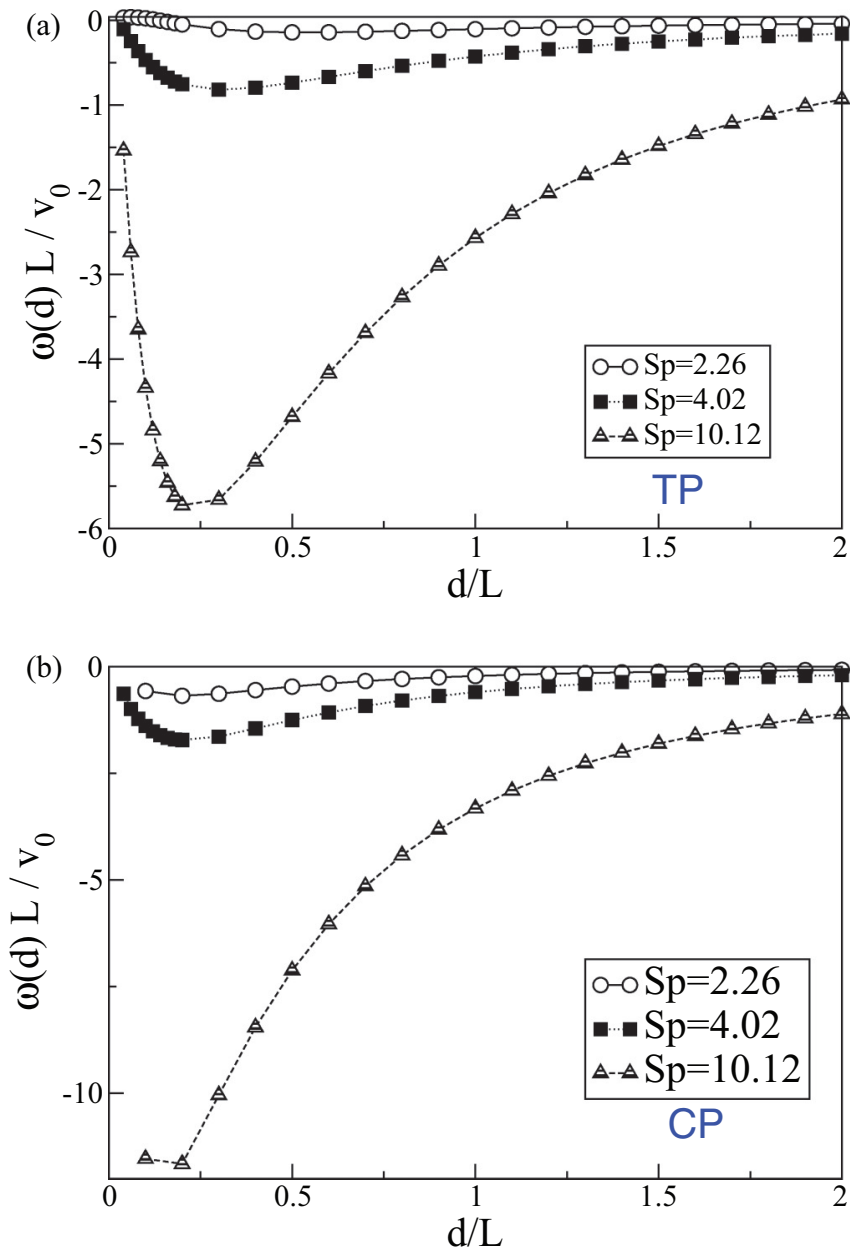

FIG. 6. (Color online) Turning angular velocity $\omega$ in the shorttime regime as a function of distance for intrinsic swimmers (a) transversal in phase (TP) and (b) coplanar in phase (CP), for different Sp numbers, at $t=\tau_{c}$, where $\tau_{c}=L \xi_{\perp} / T_{0}$ is the turning time. The angular velocity has been normalized by the propulsive velocity $v_{0}$ divided by the length of the filament $L$. The amplitude for this parameter value is $A(d \rightarrow \infty)=0.01 L$. The case of antiphase beating (not shown) follows the same behavior.

all situations, for large distances, the angular velocity always decreases algebraically as $\omega(d) \sim d^{-2}$. This is consistent with the filaments experiencing each other asymptotically as oscillating quadrupoles.

\section{Long-time turning}

As a result of the initial filament attraction, at longer times intrinsic swimmers rotate as they approach, leading to an acceleration in the change of the relative angle $\theta$ (see Fig. 7). This behavior is quite general for both transverse and coplanar beating. For the configurations analyzed, in which the typical distance between filaments spans the range $d / L=1-5$, we see that before filaments can collide they start to repel each other, relaxing toward a more parallel configuration. In all cases this change in behavior happens for a minimal relative distance less than $L$. A remaining positive and small value of $\theta$ is observed for coplanar motion, leading to a repulsion of the two filaments. For transverse motion, $\theta$ always remains

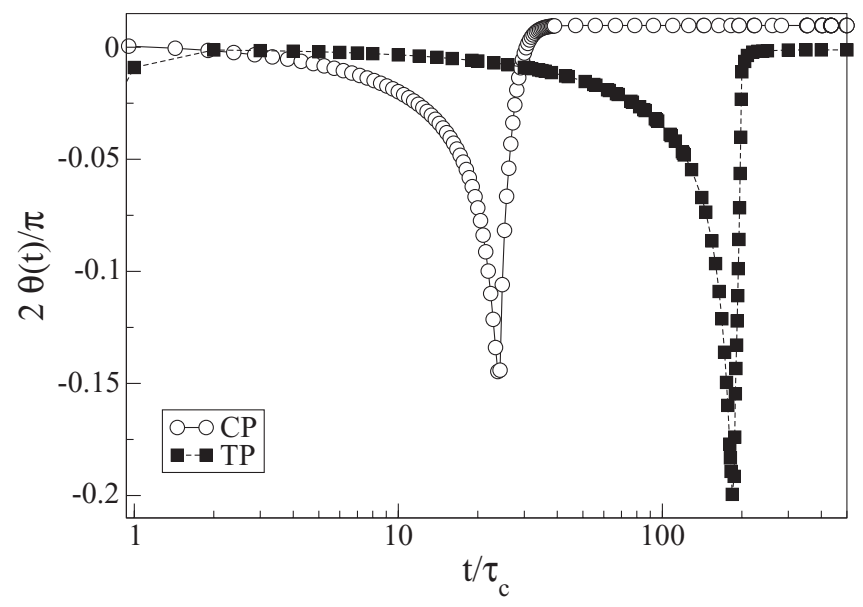

FIG. 7. Long-time turning regime: turning angle $\theta$ as a function of time for transverse bending swimmers for in-phase beating, in coplanar (CP) and transverse (TP) swimmer configurations for $\mathrm{Sp}=2.26$ and $A(d \rightarrow \infty)=0.01 L$, where $\tau_{c}=L \xi_{\perp} / T_{0}$ is the turning time. In the two cases we subtract the intrinsic rotation when computing $\theta$.

negative. This suggests the possibility of cyclic motion in which filaments experience consecutive periods of attraction and repulsion, spanning a finite set of relative distances. As described in the Introduction, a single flagellum exhibits intrinsic rotation. To assess the relevance of such intrinsic motion in the interaction with a neighbor, we have modified the wavelength of the torque wave. However, the intrinsic rotation does not significantly modify the rate of approach and repulsion of the comoving filaments. Clearly, hydrodynamic cooperativity plays the central role in determining how filaments approach or move away from each other.

\section{COOPERATIVE MOTION OF ACTUATED SWIMMERS}

In contrast to the case of intrinsic swimmers, actuated swimmers are subject to a net instantaneous acceleration and to localized stresses. Making use of the simple actuated mechanism described in Sec. II B, we briefly discuss the impact that local stresses have on cooperative swimming.

The local acceleration induced by the external field, even if, on average, actuated swimming is force-free, leads to a stronger coupling between the two filaments. This is quantified by the slower algebraic decay of the filament velocity as a function of the pair distance. For actuated swimmers we observe generically that swimming velocities decay asymptotically as $d^{-2}$. As discussed for intrinsic swimmers, this algebraic decay is weak in magnitude and the relevant hydrodynamic cooperative interactions develop at intermediate and short distances. Typically this is at separations smaller than the filament size $d<L$, where the feedback between hydrodynamic and bending stresses depends on the detailed filament morphology.

Figure 8(a) shows the velocities for parallel and coplanar filaments. One can appreciate the change in dependence from the asymptotic algebraic decay, which enhances (hinders) filament velocity for in-phase (antiphase) beating, to the short-distance dependence, in which parallel beating always increases the filament velocity. For transversal swimmers the 

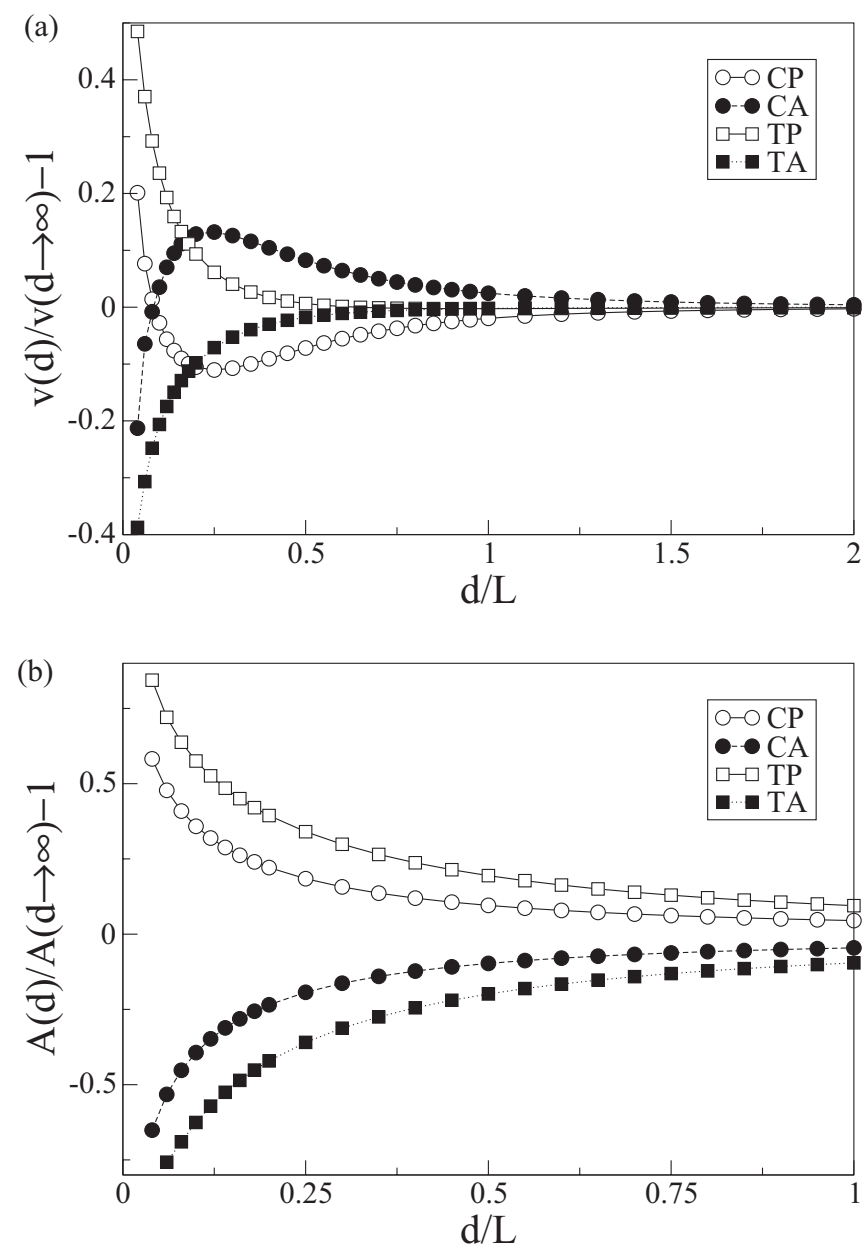

FIG. 8. (a) Velocity and (b) amplitude as a function of $d$, for $\mathrm{Sp}=2.26$ and $A(d \rightarrow \infty)=0.01 L$, when actuated swimmers move in the same plane and in parallel planes and they are driven in phase or in antiphase.

speed variation is monotonic as a function of $d$. However, for coplanar swimmers, in-phase swimming is favorable only when they are sufficiently close $d<0.1 L$. This dependence illustrates that, as opposed to intrinsic swimming, peristaltic motion is not efficient for localized forcing. This beating mode is efficient only at intermediate distances, as Fig. 8(a) shows. The variation in swimming speed is higher in transversal actuated swimmers than in coplanar actuated swimmers, but at the same time the gain in speed is in both cases higher than for transversal intrinsic swimmers and much smaller than for coplanar intrinsic swimmers. At short separations, the short-time filament velocity decreases exponentially with the pair separation, as shown in Fig. 4. As observed for intrinsic swimmers, the characteristic length $\beta$ decreases with the filaments' flexibility. However, $\beta$ now decreases monotonically with $\mathrm{Sp}$ because bending stresses are localized around the forced head and their penetration length along the filament decreases as flexibility increases. For intrinsic swimmers the whole filament length is always subject to bending stresses, regardless of its flexibility, favoring a stronger response to the hydrodynamic stresses induced by the neighbor.

In actuated swimmers, for both geometries, the amplitude increases when filaments beat in phase and decrease when out of phase, the opposite of what happens for intrinsic swimmers [Fig. 8(b)]. The localized stresses in the head of the filaments for actuated swimmers lead to a strong correlation between the motion of the actuated beads and the symmetry of the applied forces. Accordingly, the variation in beating amplitude is stronger for actuated swimmers because of the localization of the applied driving. This is higher for coplanar than for transversal beating. Finally, the localized bending stress distribution in actuated swimmers does not modify the initial approach of a swimming pair as it does for intrinsic swimmers (see Sec. III B).

\section{EFFICIENCY OF COOPERATIVE SWIMMING}

Cooperative swimming alters the mean propulsive velocity due to the motion of the fluid around it induced by the neighboring filament. If the mean flow moves in the same direction in which the filament self-propels, we expect that not only does the filament velocity increase, but the energy required will correspondingly decrease. To quantify the efficiency of cooperative swimming we focus on intrinsic swimmers, although analogous results are obtained for pairs of actuated filaments. We have considered two alternative indicators to assess the relevance of hydrodynamic cooperativity to clarify the relevance of the pair geometry and relative beating.

\section{A. Total efficiency}

A natural means for quantifying the relative efficiency of a pair of swimming filaments is the power $P_{T}$ supplied by the traveling wave generated by the internal mechanism that drives the flagellum motion. This is characterized by the bead driving $\mathbf{F}_{i}^{e}$ as a function of the separation

$$
P_{T}(d)=\frac{1}{N} \sum_{i=1}^{N_{b}} \mathbf{F}_{i}^{e} \cdot \mathbf{v}_{i},
$$

where $N_{b}$ is the number of beads composing the chain and $\mathbf{v}_{i}$ is the velocity of the bead $i$.

Since we are interested in the role that the hydrodynamic coupling between the two filaments has on the system efficiency, in Fig. 9(a) we plot the ratio between the power input at a given distance $d$ against the power supplied by the traveling wave for an isolated filament. The plots show that both the geometry and the relative beating can either promote or hinder the swimming efficiency and also indicate that hydrodynamics leads to significant changes in the power exerted by the internal drive mechanism at small filament separations. This is consistent with previous analyses for coplanar beating $[4,51]$.

We should also account for the fact that the mean swimming velocity changes as the filaments approach each other. Accordingly, we have also analyzed the quantity $E_{T}(d) \equiv$ $P_{T}(d) L / v(d)$, i.e., the work performed by the traveling wave to displace the filament a distance of its length $L$. The corresponding plots, shown in the inset of Fig. 9(a), show the same trends described for the power $P_{T}$. Namely, at small distances, the total efficiency increases (decreases) for in-phase motion and decreases (increases) for antiphase motion if swimmers are coplanar (transversal). The behavior is radically different from the swimming velocity (Fig. 3), but coincides with the fact 

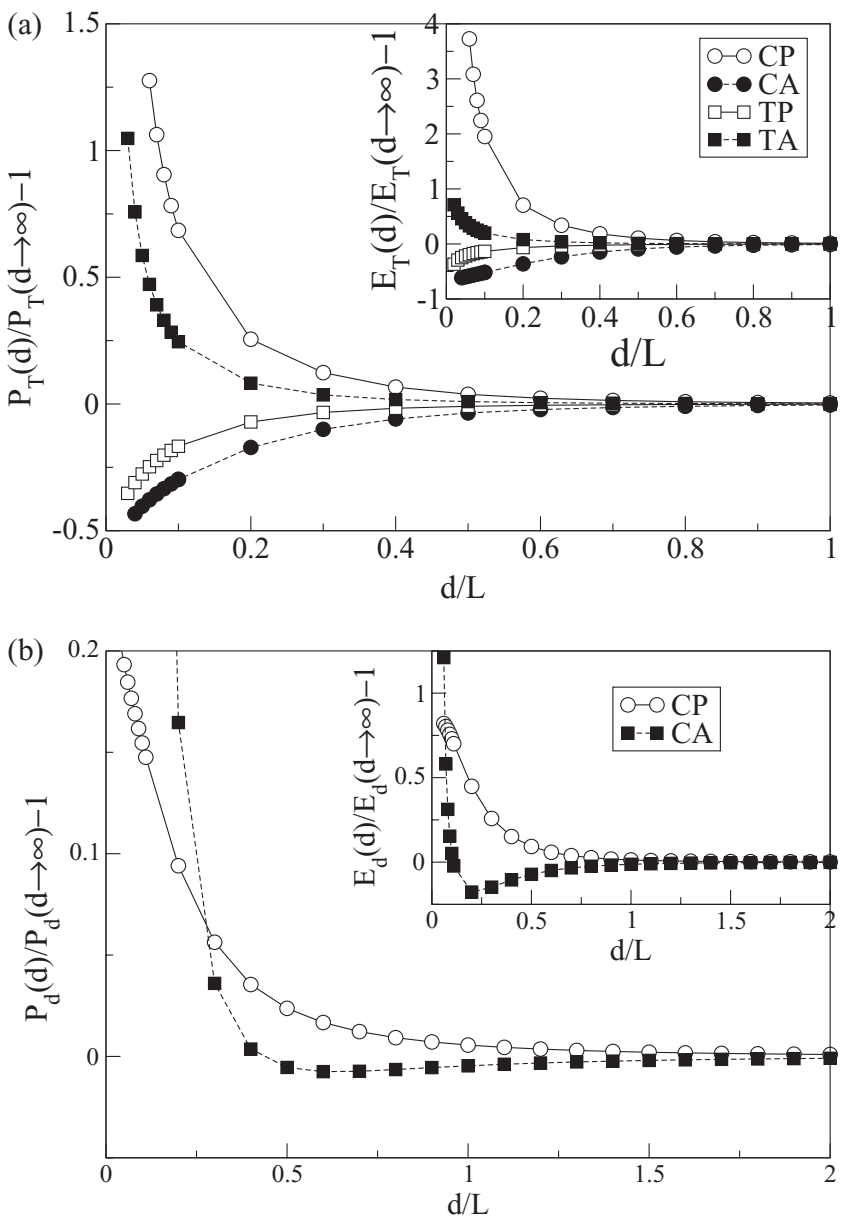

FIG. 9. (a) Total efficiency of coplanar and transversal intrinsic swimmers, at small-amplitude driving $A(d \rightarrow \infty)=0.01 L$, for $\mathrm{Sp}=2.26$. The inset shows the energy consumed after moving a distance $L$ for both geometries. (b) Dissipative efficiency of coplanar semiflexible swimmers when driven at small amplitudes $A(d \rightarrow \infty)=0.01 L$, for $\mathrm{Sp}=2.26$. The inset shows the energy dissipated after moving a distance $L$ for coplanar swimmers.

that the change of efficiency due to the neighboring filament is higher for coplanar swimmers. Moreover, the figure shows that the relative effects of hydrodynamic interactions are larger for the energy to displace the filament by its length than for the dissipated power.

\section{B. Dissipative efficiency}

As an alternative measure of the efficiency, we have also analyzed the dissipated power associated with the filament motion, quantified by the dissipative force the fluid exerts on the swimmers. Accordingly, we introduce the power spent working against the fluid drag

$$
P_{d}(d)=\frac{1}{N} \sum_{i=1}^{N_{b}}\left(\gamma_{0} \mathbf{v}_{i}-\mathbf{v}_{i}^{H}\right)^{2} .
$$

Figure 9(b) shows $P_{d}$ for a pair of swimmers at distance $d$, relative to the intrinsic dissipated power of an isolated filament for the particular case of coplanar swimmers, where we have seen the largest sensitivity to hydrodynamic cooperation in the power input $P_{T}$. The dissipated power increases as the two filaments approach regardless of the relative motion of the two filaments. The inset of Fig. 9(b) shows $E_{d}(d) \equiv P_{d}(d) L / v(d)$, the energy dissipated by the swimmer as it displaces its own size $L$, as a function of the relative distance $d$. This dissipation efficiency increases monotonically for in-phase motion as the distance decreases. However, for antiphase motion there is a characteristic distance $d \sim 0.2 L$ where the efficiency is smaller, but it is larger at smaller distances. Such behavior is different from the velocity and amplitude dependences and from the total efficiency of Fig. 9(b).

Although $P_{d}$ always increases as the separation decreases, the relative increase is smaller than that observed for $P_{T}$. Therefore, for in-phase beating cooperative swimming is more efficient than isolated swimming. The opposite is true for antiphase swimming. The variation in efficiency induced by a neighbor can be quantified through the ratio $P_{T}(d) P_{d}(\infty) / P_{d}(d) P_{T}(\infty)-1$, which is positive (negative) for in-phase (antiphase) swimmers. These results show that coupling through the fluid plays a role in inducing a significant increase in swimming efficiency if filaments can beat in a coordinated fashion. This fact suggests that synchronization may have additional benefits coming from the reduction of dissipation due to hydrodynamic cooperativity.

\section{DISCUSSION AND CONCLUSION}

We have analyzed the cooperative motion of model semiflexible swimming filaments representing biological flagella or technological microswimmers and have focused on the sources of hydrodynamic coupling for characteristic geometries, the role of driving, and their relevance in the motion of these swimmers. We have shown that the coupling between the beating velocity and the secondary flows induced by the deformed chains leads to scenarios that cannot be captured either by resistive force theory (which would fail to predict any hydrodynamic coupling between the two swimmers) or by simplified models based on longitudinal swimmers. The near flow field, controlled by the domains produced by the traveling wave associated with self-propulsion, leads to near-field interactions that differ in magnitude and sign from the predictions from the far-field asymptotic interaction among filaments. Although the far-field decay is always algebraic, the near field is significantly stronger in magnitude and displays a distinct sensitivity to both filament separation and flexibility.

The different properties of the near and far fields show that the dynamic characterization of joint swimming depends not only on the details of beating and the relative orientation of the chains, but also on filament separation. This fact might be connected to the observation that rodent spermatozoon trains do not always swim faster than individuals, but may actually be slower [14].

By comparing intrinsic and actuated swimmers, we have analyzed the impact that the stress distribution along the filament has on cooperative swimming. Actuated filaments are subject to instantaneous accelerations that induce longerranged interactions and to local bending stresses, leading to a faster decoupling for nearby filaments than those observed for intrinsic swimmers. 
We have also identified generic features of the hydrodynamic cooperativity between swimming semiflexible filaments. Specifically, frontal swimmers effectively repel each other while parallel swimmers, either coplanar or transversal, initially rotate due to their interactions and approach each other. The induced rotation ultimately leads to an effective repulsion at longer times as a result of the deformation induced by such rotation. If flagella need to keep swimming together to benefit from their cooperativity, they need to develop strategies to link with each other. This is compatible, for example, with the hooks observed in rodent spermatozoon trains.

All these results support the conclusion that hydrodynamic considerations are crucial when considering cooperativity in the propulsion of flagellated organisms and the crucial role of how such a propulsion is produced. This fact suggests that flagellated organisms may have developed strategies to benefit from such coupling either to move in the presence of other flagella or to maximize propulsive efficiency in the case of multiflagellar micro-organisms. For the latter, we have observed that hydrodynamic cooperativity and synchronization can team up and a large number of flagella swimming together change the local fields and hence their swimming velocities [37]. This is known in the case of the rotation of an ensemble of cilia leading to their synchronized motion [25]. Biological flagella tend to synchronize and then attract to swim together [20,22]. However, we predict that this strategy is not always the one that leads to a faster speed. The speed gain depends in a complex way on the geometry and the relative distance.

\section{ACKNOWLEDGMENTS}

I.Ll. and I.P. acknowledge financial support from DGCIYT of the Spanish Government (Grant No. FIS2011-22603) and DURSI (Grant No. 2009SGR-634).
[1] D. Bray, Cell Movements (Garland, New York, 1992).

[2] E. M. Purcell, Am. J. Phys. 45, 3 (1977).

[3] K. Drescher, R. E. Goldstein, N. Michel, M. Polin, and I. Tuval, Phys. Rev. Lett. 105, 168101 (2010); J. S. Guasto, K. A. Johnson, and J. P. Gollub, ibid. 105, 168102 (2010).

[4] G. I. Taylor, Proc. R. Soc. London Ser. A 209, 447 (1951).

[5] C. Brennen and H. Winet, Annu. Rev. Fluid. Mech. 9, 339 (1977).

[6] J. Higdon, J. Fluid Mech. 90, 685 (1979).

[7] K. E. Machin, J. Exp. Biol. 35, 796 (1958).

[8] C. H. Wiggins and R. E. Goldstein, Phys. Rev. Lett. 80, 3879 (1998).

[9] M. Cosentino Lagomarsino, F. Capuani, and C. P. Lowe, J. Theor. Biol. 224, 215 (2003).

[10] M. Manghi, X. Schlagberger, and R. R. Netz, Phys. Rev. Lett. 96, 068101 (2006).

[11] A. Najafi and R. Golestanian, Phys. Rev. E 69, 062901 (2004).

[12] E. A. Gaffney, H. Gadêlha, D. J. Smith, J. R. Blake, and J. C. Kirkman-Brown, Annu. Rev. Fluid Mech. 43, 501 (2011).

[13] M. Polinm I. Tuval, K. Drescher, J. P. Gollub, and R. E. Goldstein, Science 325, 487 (2009).

[14] S. Immler, H. D. M. Moore, W. G. Breed, and T. R. Birkhead, PLoS ONE 2, e170 (2007).

[15] H. Moore, K. Dvoráková, N. Jenkins, and W. Breed, Nature (London) 418, 174 (2002).

[16] M. Eisenbach and L. C. Giojalas, Nat. Rev. Mol. Cell Biol. 7, 276 (2006).

[17] G. J. Elfring and E. Lauga, Phys. Fluids 23, 011902 (2011).

[18] G. J. Elfring and E. Lauga, J. Fluid Mech. 674, 163 (2011).

[19] R. E. Goldstein, M. Polin, and I. Tuval, Phys. Rev. Lett. 103, 168103 (2009).

[20] Y. Yang, J. Elgeti, and G. Gompper, Phys. Rev. E 78, 061903 (2008).

[21] M. Reichert and H. Stark, Eur. Phys. J. E 17, 493 (2005).

[22] D. M. Woolley, R. F. Crockett, W. D. I. Groom, and S. G. Revell, J. Exp. Biol. 212, 2215 (2009).

[23] B. Bassetti, M. Cosentino Lagomarsino, and P. Jona, Eur. Phys. J. B 15, 483 (2000).
[24] S. Gueron and K. Levit-Gurevich, Proc. Natl. Acad. Sci. USA 96, 12240 (1999).

[25] A. Vilfan and F. Jülicher, Phys. Rev. Lett. 96, 058102 (2006).

[26] S. Camalet and F. Julicher, New J. Phys. 2, 24 (2000).

[27] S. Camalet, F. Jülicher, and J. Prost, Phys. Rev. Lett. 82, 1590 (1999).

[28] L. Dreyfus, J. Baudry, M. L. Roper, M. Fermigier, H. A. Stone, and J. Bibette, Nature (London) 437, 862 (2005).

[29] M. L. Roper, R. Dreyfus, J. Baudry, M. Fermigier, J. Bibette, and H. A. Stone, J. Fluid. Mech. 554, 167 (2006).

[30] J. J. Abbott, K. E. Peyer, M. Cosentino Lagomarsino, L. Zhang, L. X. Dong, I. K. Kaliakatsos, and B. J. Nelson, Int. J. Robot. Res. 28, 1434 (2009).

[31] E. Lauga, Phys. Rev. E 75, 041916 (2007).

[32] E. Lauga and T. R. Powers, Rep. Prog. Phys. 72, 096601 (2009).

[33] C. P. Lowe, Future Gener. Comput. Syst. 17, 853 (2001).

[34] M. Doi and S. F. Edwards, The Theory of Polymer Dynamics (Oxford University Press, New York, 1986).

[35] M. C. Lagomarsino, I. Pagonabarraga, and C. P. Lowe, Phys. Rev. Lett. 94, 148104 (2005).

[36] A. G. Bailey, C. P. Lowe, I. Pagonabarraga, and M. C. Lagomarsino, Phys. Rev. E 80, 046707 (2009).

[37] I. Llopis, M. Cosentino Lagomarsino, I. Pagonabarraga, and C. P. Lowe, Comput. Phys. Commun. 179, 150 (2008).

[38] I. Llopis, I. Pagonabarraga, M. Cosentino Lagomarsino, and C. P. Lowe, Phys. Rev. E 76, 061901 (2007).

[39] J. Gray and G. J. Hancock, J. Exp. Biol. 32, 802 (1955).

[40] M. A. Sleigh, Cilia and Flagella (Academic, London, 1974).

[41] B. Friedrich and F. Jülicher, Proc. Natl. Acad. Sci. USA 104, 13256 (2007).

[42] E. Gauger and H. Stark, Phys. Rev. E 74, 021907 (2006).

[43] E. E. Keaveny and M. R. Maxey, J. Fluid Mech. 598, 293 (2008).

[44] R. Livanovičs and A. Cēbers, Phys. Rev. E 85, 041502 (2012). 
[45] C. H. Wiggins, D. Riveline, A. Ott, and R. E. Goldstein, Biophys. J. 74, 1043 (1998)

[46] C. P. Lowe and S. de Leeuw, in Proceedings of the Fifth Annual Conference of the Advanced School for Imaging and Computing, Heijen, 1999, edited by A. Hoekstra and J. F. M. Tonino (Technische Universiteit Delft, Delft, 1999), pp. 279-287.

[47] D. J. Earl et al., J. Chem. Phys. 126, 064703 (2007).
[48] G. P. Alexander, C. M. Pooley, and J. M.Y eomans, J. Phys.: Condens. Matter 21, 204108 (2009).

[49] Y. Yang, V. Marceau, and G. Gompper, Phys. Rev. E 82, 031904 (2010).

[50] C. M. Pooley, G. P. Alexander, and J. M. Yeomans, Phys. Rev. Lett. 99, 228103 (2007).

[51] L. J. Fauci, J. Comput. Phys. 86, 294 (1990). 